\title{
Editorial
}

\section{Análisis de los indicadores generales de Publindex 2002 - 2010}

\author{
Gabriel Peña Rodríguez ${ }^{1}$
}

$\mathrm{P}$ ublindex, es un sistema de información del Departamento Administrativo de Ciencia, Tecnología e Innovación (COLCIENCIAS), encargada en el ámbito Nacional de la Indexación y la Homologación de revistas especializadas en Ciencia, Tecnología e Investigación $(\mathrm{CT}+\mathrm{I})$. Dependencia, que administra la Base Bibliográfica Nacional (BBN), o listado de las revistas Colombianas indexadas, y las revistas extranjeras homologadas, según los criterios de existencia, calidad científica y visibilidad establecidos en sus políticas.

Como herramienta de apoyo a los editores e investigadores del País, Publindex a mediados del mes de octubre de 2011, teniendo en cuenta la información de la BBN en el periodo de 2002 a 2010, presentó indicadores de las revistas nacionales por categorías (A1, A2, B y C), áreas de la ciencia según la Organización para la Cooperación y Desarrollo Económico (OCDE) y tipo de institución editora. De este informe, se aprecia un incremento del 308 $\%$, pasando de 91 revistas indexadas en el 2002 a 372 para el 2010. Se observa una disminución en el porcentaje de revistas indexadas en la categoría C (81,3 \% en el 2002 a 56,7\% en el 2010), lo cual contrasta con el aumento de los porcentajes de las revistas indexadas para la categoría B (7,7 \% en 2002 a 17,7 \% en 2010), categoría A2 (9,9\% en 2002 a 19,4 \% en 2010) y categoría A1 (1,1\% en 2002 a 6,2\% en 2010), reflejándose los esfuerzos de los editores por cumplir las exigencias de Publindex para ascender a otras categorías .

De los datos reportados por áreas de la ciencia según la OCDE, se infiere que las dos áreas que tienen la mayor cantidad de revistas indexadas en este periodo, son las ciencias sociales (29 al 38\%) seguidas de las ciencias médicas y de la salud (17 al 22\%). Las áreas que presentan el menor número de revistas son ciencias agrícolas (5 al $8 \%$ ), y las multidisciplinarias (2 al $6 \%$ ), mientras que el área de ingeniería y tecnología, reporta un porcentaje que oscila entre el 9 al 13 \%. Lo anterior indica, que en el País, las dos áreas donde hay mayor cantidad de grupos de investigación son las ciencias sociales, y las ciencias médicas y de la salud, información que se pudo confirmar, al consultar la plataforma ScienTi-COLCIENCIAS, donde efectivamente estas dos áreas son las que tienen el mayor número de grupos de investigación como actividad principal, 1945 y 769 grupos respectivamente.

$\mathrm{Al}$ analizar las instituciones editoras de las revistas se observa que las Instituciones de Educación Superior (IES) privadas han ido incrementado el porcentaje de edición de las revistas indexadas, pasando del 29\% para el año 2002, al $49 \%$ en el 2010. Mientras que las IES públicas han decrecido en el número de revistas indexadas, pasado del 52\% en el 2002, al 40\% para el año 2010. Habría que revisar, si este incremento de las revistas editadas por las IES privadas, se da en las de mayor categoría (A1), información que no se aprecia en el listado publicado por Publindex para este periodo. Sin embargo, para las EIS públicas, es un indicador que debe servir en la toma de decisiones de investigación y publicación, a los gestores de investigación (Vicerrectores), editores e investigadores.

En general estos indicadores, han permitido a Publindex trazar algunas políticas que serán aplicadas en los próximos años, en las cuales se destacan: medir la visibilidad de los Sistemas de Indexación y Resumen (SIR) para todas las revistas desde las categorías C hasta las A1; las revistas indexadas en las categorías A1 y A2, deben estar en los cuartiles uno y dos (Q1 y Q2) del Journal Citation Reports (JCR) y del ISI-SCI (Institute for Scientific Information - Science Citation Index), o de Scimago-SCOPUS; la categoría B, se pretende dividir en B1 y B2, según los SIR donde sean visibles dichas revistas, para la B1, la revista debe estar indexada en un índice regional (Scielo-Redalyc) o el cuartil cuatro (Q4) del ISI o SCOPUS, mientras que la B2, debe pertenecer a dos Bases Bibliográficas con Comité de Selección (BBCS); para las revistas en la categoría C, se incrementa el número de artículos de investigación e innovación de 9 a 12 por año, y el $75 \%$ de los artículos deben ser externos a la institución editora; todas las revistas deben tener pagina web y adoptar el Open Journal System (OJS), que es un sistema (software libre) de administración y publicación de revistas y documentos seriados en Internet.

${ }^{1}$ Doctor en ingeniería

de materiales. Profesor adscrito al departamento de física-UFPS. Investigador CIMAC-UFPS. 